\title{
Optimal Load Dispatch of Gas Turbine Power Generation Units based on Multiple Population Genetic Algorithm
}

\author{
Hua Xiao, Cheng Yang, Jie Wu, Xiaoqian Ma \\ College of Electric Power, South China University of Technology, Guangzhou 510640, China \\ Email: xhua2008@163.com, chyang1@scut.edu.cn, jiewu7@163.com, epxqma@scut.edu.cn
}

Received 2013

\begin{abstract}
In this paper, a multiple population genetic algorithm (MPGA) is proposed to solve the problem of optimal load dispatch of gas turbine generation units. By introducing multiple populations on the basis of Standard Genetic Algorithm (SGA), connecting each population through immigrant operator and preserving the best individuals of every generation through elite strategy, MPGA can enhance the efficiency in obtaining the global optimal solution. In this paper, MPGA is applied to optimize the load dispatch of $3 \times 390 \mathrm{MW}$ gas turbine units. The results of MPGA calculation are compared with that of equal micro incremental method and AGC instruction. MPGA shows the best performance of optimization under different load conditions. The amount of saved gas consumption in the calculation is up to $2337.45 \mathrm{~m}^{3} \mathrm{~N} / \mathrm{h}$, which indicates that the load dispatch optimization of gas turbine units via MPGA approach can be effective.
\end{abstract}

Keywords: Gas turbine generation units; Load dispatch; MPGA; Optimization

\section{Introduction}

The gas turbine centered gas-steam combined cycle with high thermal efficiency, low pollution discharge, short building time and advanced start-stop performances is well-accepted and fast-growing in electricity generation industry. In this situation, it is important to improve the efficiency of gas turbine power plants. Load dispatch is an essential method to improve the economic efficiency which makes the load dispatch optimization a key point. In mathematics, the problem of optimizing load dispatch is a complex high-dimensional nonlinear problem containing integer and continuous variables [1-4].

With the development of computer technology and artificial intelligence, modern intelligent algorithms show great advantages in solving load dispatch problems, which mainly includes simulated annealing, artificial neural network, ant colony optimization, genetic algorithm and chaos optimization [5,6]. Among them, genetic algorithm has a fast development due to fewer limits and requirements for continuous or differentiable objective function [7]. Moreover, it shows of strong robustness characteristics, global optimization and parallel computing in solving nonlinear problem, which makes it widely applied in optimal operation of power system.

Therefore, this paper is to focus on proposing an efficient algorithm to solve the the problem of optimal load dispatch of gas turbine generation units. A multiple population genetic algorithm is adopted to optimize the calculation of load dispatch of a gas-turbine plant in this paper [8-10], which shows a better find-best ability to solve the problem.

\section{Optimal Load Dispatch Model of Gas Turbine Generation Units}

Binomial expression is usually adopted to fit the characteristic curve of gas consumption of gas-turbine generation units. The function of gas consumption has the form as follow.

$$
f_{i}\left(P_{i}\right)=\left(a_{i} P_{i}^{2}+b_{i} P_{i}+c_{i}\right)
$$

where $a_{i}, b_{i}, c_{i}$ are the gas consumption characteristic coefficients of each unit; $f_{i}$ is the gas consumption of each unit; $P_{i}$ is the load of each unit. Suppose the whole plant has $\mathrm{n}$ units which can be put into operation and the total load demand is $P_{D}$. So the goal of the load dispatch is to minimize the total gas consumption by distributing the load to each of the $\mathrm{n}$ units reasonably according to their gas consumption characteristics. The load dispatch model can be described as follows.

$$
\left\{\begin{array}{l}
\min F=\sum_{i=1}^{n} U_{i} \bullet f_{i}\left(P_{i}\right) \\
\text { s.t. } \quad \sum_{i=1}^{n} U_{i} P_{i}=P_{D} \\
\boldsymbol{P}_{i \min } \leq \boldsymbol{P}_{i} \leq \boldsymbol{P}_{i \max } \\
\sum_{i=1}^{n} \boldsymbol{U}_{i} \boldsymbol{P}_{i \max } \geq \boldsymbol{P}_{D} \\
\sum_{i=1}^{n} \boldsymbol{U}_{i} \boldsymbol{P}_{i \min } \leq \boldsymbol{P}_{D}
\end{array}\right.
$$


where, $U_{i}$ is the state of unit. 0 and 1 denote closing down and running of a unit, respectively; $P_{i \min }$ is the minimum load permitted; $P_{i \max }$ is the maximum load permitted; $P_{D}$ is the total load demand.

It should be noted that the performance of Gas Turbine Combined Cycle (GTCC) is influenced by the ambient conditions such as temperature, pressure and so on. Therefore the performance should have been revised according to the ambient consitions. The influence of ambient conditions is not discussed in this paper for space limitations. Generally speaking, the pressure of a certain place varies so slightly that its influence can be ignored. And every $1^{\circ} \mathrm{C}$ depression in inlet air temperature results in about $0.45 \%$ power increase of GTCC and slight variation in heat consumption rate $[11,12]$.

\section{The Model of Multiple Population Genetic Algorithm}

\subsection{Overviews of the Algorithm}

Genetic algorithm derives from computer simulation study of biological systems. It is a self-adaption optimization algorithm that simulates heredity and evolution of biology in environment. The basic idea of using genetic algorithm is expressing the $\mathrm{n}$ units as real vectors and using random selection or other methods to generate "initial population". Then individuals in the group of each generation proceed selection, cross and mutation by certain probability. According to survival-of-the-fittest mechanism, it adopts stepwise iteration method until the optimal load dispatch results are obtained. Genetic algorithm has less special requirements for objective function. It can find global optimal solutions in theory. It also can provide many optional solutions and fits for parallel processing. Multi-population genetic algorithm (MPGA) is based on standard genetic algorithm but it has a better ability of global search and can overcome the problem of premature convergence compared to standard genetic algorithm. In MPGA, many populations evolve simultaneously which adopt the same methods as standard genetic algorithm (SGA) and realize coevolution through immigrant operator connecting different populations. Elite operator is adopted to preserve the best individual of each generation, which judges the convergence [13, 14]. In all, MPGA mainly includes such steps as: initial populations, selection, heredity, variation, migration and artificial selection. The structure of MPGA is shown in Figure 1.

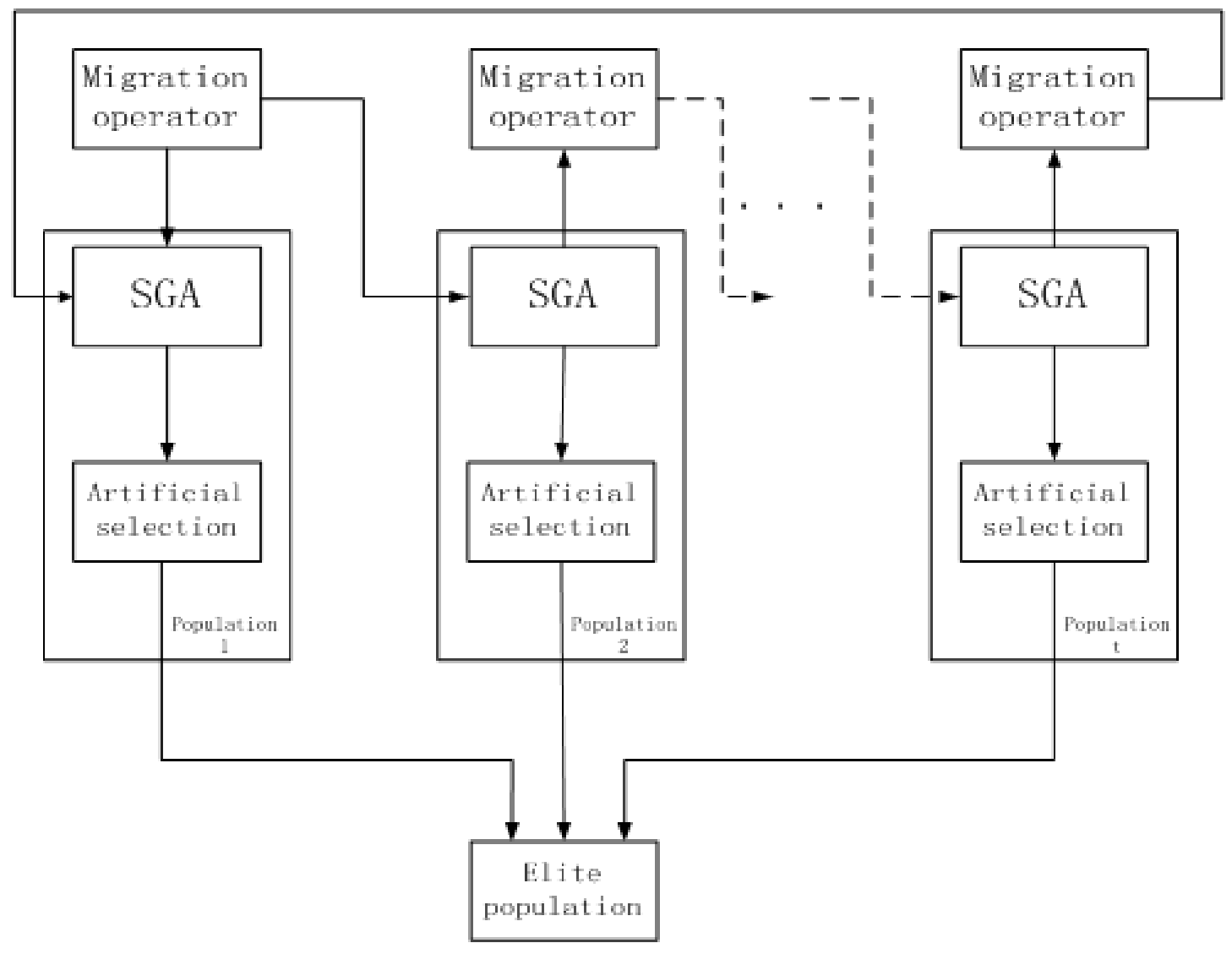

Figure 1. Structure diagram. 


\subsection{Initial Populations}

Feasible solutions of load dispatch are coded into chromosomes or individuals. Generally the coding methods include bit string coding, grey coding, real coding, multi parameter coding and etc. For load dispatch problems, the real number coding needs no value conversion and we can directly carry out genetic manipulation on phenotype of solutions. Therefore we choose real coding method in this paper. Every chromosome is a real vector.

Then, $\mathrm{N}$ sets of data representing load dispatch solutions are generated in random. Every set of data is an individual. MPGA generate t populations and each population consists of $\mathrm{N} / \mathrm{t}$ individuals. MPGA begins to evolve with the $\mathrm{N}$ data.

\subsection{Fitness Function}

Fitness function is the function that needs to be optimized. It is the criteria to judge whether an individual is good or not. Fitness functions are generally converted from objective functions. For the problem of optimal load dispatch, the smaller the value of fitness function, the better the fitness of an individual. For load dispatch of gas-turbine generation units, the fitness function is described as follows.

$$
g=\sum_{i=1}^{n} U_{i} \bullet f_{i}\left(P_{i}\right)+C\left(\sum_{i=1}^{n} U_{i} P_{i}-P_{D}\right)^{2}
$$

where penalty factor $C$ usually takes a larger value in the process of optimization[7]. It will lead to a large value to the objective function if the solution does not satisfy the restriction, so that the solution will be eliminated.

\subsection{Selecting Operation}

Selection is to select those individuals whose fitness functions values are small to become parents and generate a new population to reproduce the individuals of next generation. The individuals of oversize fitness function value will be eliminated because of maladjustment.

Selecting operation involves roulette method, championship method and etc. In this paper roulette method is adopted. The probability to enter next generation for every individual equals to the rate of its own fitness and the sum of all the individuals' fitness. The bigger the fitness value, the greater probability for an individual to be selected and the greater probability to pass into next generation are. So the probability of being selected for individual $\mathrm{j}$ is:

$$
p_{j}=\frac{F_{j}}{\sum_{j=1}^{n} F_{j}}
$$

where $F_{j}$ is the fitness of $j, n$ is the amount of individuals in one population.

\subsection{Crossover Operation}

Cross is to select two individuals from parent generation, by the crossover of chromosomes, to generate a new good individual. Real crossover method is used in this paper. The cross operation method of No. $\mathrm{m}$ chromosome $\mathrm{a}_{\mathrm{m}}$ and No. $\mathrm{n}$ chromosome in position $\mathrm{k}$ is described as follows.

$$
\left\{\begin{array}{l}
a_{m k}=a_{i k}(1-b)+a_{n k} b \\
a_{n k}=a_{i k}(1-b)+a_{m k} b
\end{array}\right.
$$

where $b$ is a random number in region $[0,1]$.

\subsection{Mutation Operation}

Mutation is to randomly select one point of an individual from parent generation and mutate the point so as to generate a random individual. The mutation operation method of No. $\mathrm{j}$ gene of No. i individual is described as follows[7].

$$
a_{i j}=\left\{\begin{array}{l}
a_{i j}+\left(a_{i j}-a_{\max }\right) \bullet f(g), r \geq 0.5 \\
a_{i j}+\left(a_{\min }-a_{i j}\right) \bullet f(g), r<0.5
\end{array}\right.
$$

where amax is the upper bound of gene; aij, amin is the lower bound of gene $\mathrm{a}_{\mathrm{ij}} ; \quad f(g)=r_{2}\left(1-g / G_{\max }\right)^{2}, \mathrm{r}_{2}$ is a random number, $g$ is current iterations, Gmax is the maximum evolution time, $r$ is a random number in region $[0,1]$.

\subsection{Elite Strategy}

In MPGA, different populations develop through different operation parameters to achieve different search targets. Then it realizes multiple populations' co-evolution through the migration operator. The migration operator compares the best individuals of each population and substitutes the worst individuals of each population by globally optimal individual. Then by artificial selection operator, optimal individuals of each population and generation are recorded as the criterion of algorithm convergence[7].

\section{Simulation}

In [6] the author uses equal micro incremental to optimize the load dispatch of $3 \times 390 \mathrm{MW}$ gas turbine generation units. In this paper, the gas consumption characteristic equations from [6] are used for the calculation of the MPGA.

According to paper [6] the characteristic coefficient of gas consumption function and bound constraints are shown in Table 1. 
Table 1. Gas consumption character coefficient and limit of load.

\begin{tabular}{|c|c|c|c|c|c|}
\hline date & unit & $\mathbf{a}_{\mathbf{i}}$ & $\mathbf{b}_{\mathbf{i}}$ & $c_{i}$ & {$\left[p_{\min }, \mathbf{p}_{\max }\right]$} \\
\hline 7.25 & 1 & 0.11837 & 84.002 & 16725 & {$[234,390]$} \\
\hline 7.25 & 2 & 0.12583 & 75.695 & 18274 & {$[234,390]$} \\
\hline 10.26 & 1 & 0.058427 & 117.29 & 11904 & {$[234,390]$} \\
\hline 10.26 & 3 & 0.12681 & 71.863 & 17860 & {$[234,390]$} \\
\hline
\end{tabular}

Take total load $\mathrm{P}_{\mathrm{D}}=505 \mathrm{MW}$ on July 25, 2007 as an example. Fitness function is described as follow.

$$
g=\sum_{i=1}^{3} U_{i} \cdot f_{i}\left(P_{i}\right)+C\left(\sum_{i=1}^{3} U_{i} P_{i}-P_{D}\right)^{2}
$$

The units in operation are just $1 \#$ and $2 \#$, so $\mathrm{U}_{1}=\mathrm{U}_{2}=1$, $\mathrm{U}_{3}=0$. Take 100 for the penalty factor $\mathrm{C}$.

$$
\left\{\begin{array}{l}
f_{1}\left(P_{1}\right)=0.11837 P_{1}^{2}+84.002 P_{1}+16725 \\
f_{2}\left(P_{2}\right)=0.12583 P_{2}^{2}+75.695 P_{2}+18274
\end{array}\right.
$$

In this multiple population genetic algorithm model, the amount of the populations is set to 10 . Cross probability pc of each population randomly generates between 0.7 and 0.9 , Mutation probability pm randomly generates between 0.01 and 0.05 . The size of population is set to 40.
Through the optimization of MPGA, the minimum value of fitness function reaches 90833.33, with the load of $1 \#$ unit $242.84 \mathrm{MW}$ and $2 \#$ unit $261.46 \mathrm{MW}$. The total gas consumption is $90771.60 \mathrm{~m}^{3} \mathrm{~N} / \mathrm{h}$. The evolution process of this calculation is shown as Figure 2.

In a similar way, all the results of load dispatch calculation are obtained and compared with results of other methods, as shown in Table 2 and Table 3.

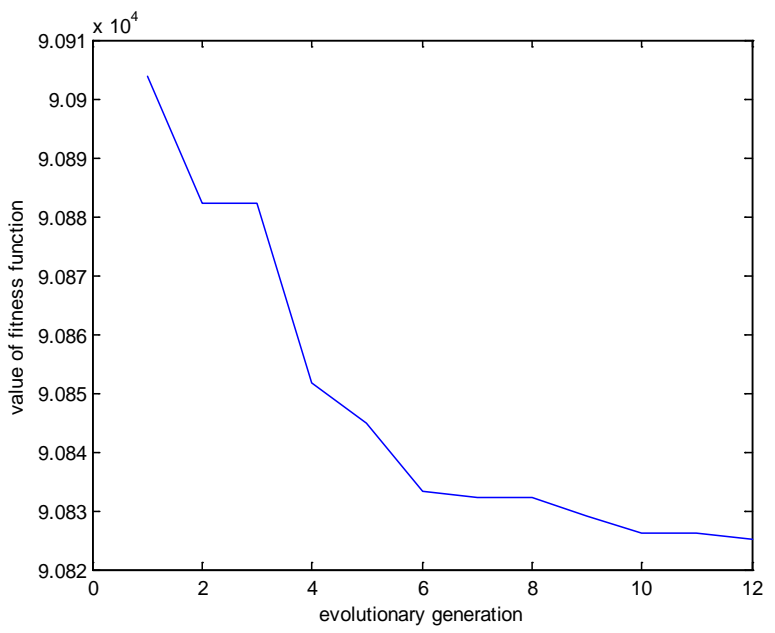

Figure 2. Evolution process.

\begin{tabular}{|c|c|c|c|c|c|c|c|}
\hline $\begin{array}{l}\text { Load } \\
\text { MW }\end{array}$ & $\begin{array}{c}\mathbf{P}_{1} \\
\text { (MPGA) } \\
\text { MW }\end{array}$ & $\begin{array}{c}\mathbf{P}_{2} \\
\text { (MPGA) } \\
\text { MW }\end{array}$ & $\begin{array}{l}\text { 1\# } \\
\text { gas consumption } \\
\left(\text { MPGA) } \mathrm{m}^{3} \mathrm{~N} / \mathrm{h}\right.\end{array}$ & $\begin{array}{l}2 \# \\
\text { gas consumption } \\
\text { (MPGA) } \mathrm{m}^{3} \mathrm{~N} / \mathrm{h}\end{array}$ & $\begin{array}{l}\text { Total gas consump- } \\
\text { tion (MPGA) } \mathbf{m}^{3} \mathrm{~N} / \mathrm{h}\end{array}$ & $\begin{array}{c}\text { Equal micro } \\
\text { incremental method } \\
\mathbf{m}^{3} \mathrm{~N} / \mathrm{h}\end{array}$ & $\begin{array}{c}\text { AGC } \\
\mathbf{m}^{3} \mathbf{N} / \mathbf{h}\end{array}$ \\
\hline 505 & 242.84 & 261.46 & 44104.47 & 46667.12 & 90771.60 & 90870.67 & 98274.51 \\
\hline 542 & 261.87 & 279.39 & 46839.93 & 49244.56 & 96084.49 & 96192.57 & 98427.58 \\
\hline 600 & 291.00 & 307.00 & 51193.27 & 53371.72 & 104564.99 & 104871.10 & 104935.59 \\
\hline 660 & 322.68 & 336.52 & 56155.73 & 57996.59 & 114152.32 & 114280.70 & 117339.50 \\
\hline 700 & 343.26 & 355.91 & 59506.76 & 61153.74 & 120660.50 & 120797.70 & 122443.95 \\
\hline
\end{tabular}

Table 2. Load dispatch results of 1\#,2\# by different methods On July 25,2007.

\begin{tabular}{|c|c|c|c|c|c|c|c|}
\hline $\begin{array}{l}\text { Load } \\
\text { MW }\end{array}$ & $\begin{array}{c}\mathbf{P}_{1} \\
\text { (MPGA) } \\
\text { MW }\end{array}$ & $\begin{array}{c}\mathbf{P}_{3} \\
\text { (MPGA) } \\
\text { MW }\end{array}$ & $\begin{array}{l}1 \# \\
\text { gas consumption } \\
\text { (MPGA) } \mathrm{m}^{3} \mathrm{~N} / \mathrm{h}\end{array}$ & $\begin{array}{c}3 \# \\
\text { gas consumption } \\
\text { (MPGA) } \mathrm{m}^{3} \mathrm{~N} / \mathrm{h}\end{array}$ & $\begin{array}{l}\text { Total gas consumption } \\
\text { (MPGA) } \mathrm{m}^{3} \mathrm{~N} / \mathrm{h}\end{array}$ & $\begin{array}{c}\text { Equal micro } \\
\text { incremental method } \\
\mathbf{m}^{3} \mathrm{~N} / \mathrm{h}\end{array}$ & $\begin{array}{l}\text { AGC } \\
\mathbf{m}^{3} \mathbf{N} / \mathbf{h}\end{array}$ \\
\hline 491 & 234.39 & 256.1 & 42560.04 & 43008.05 & 85563.06 & 87114.16 & 88041.39 \\
\hline 500 & 234.10 & 265.26 & 42563.81 & 43606.07 & 86169.88 & 88507.33 & 87778.98 \\
\hline 600 & 290.00 & 309.16 & 50831.87 & 50820.74 & 101652.61 & 103229.10 & 103538.45 \\
\hline 651 & 322.78 & 327.46 & 55850.32 & 54654.14 & 110504.46 & 111009.30 & 119029.83 \\
\hline 701 & 356.27 & 343.93 & 61106.92 & 58492.97 & 118569.89 & 118737.80 & 119029.83 \\
\hline
\end{tabular}

Table 3. Load dispatch results of 1\#,3\# by different methods On October 26,2007. 
According to the results above, MPGA has an obvious decrease of total gas consumption compared to the equal micro incremental method and AGC instruction. When the total load demand is 500MW on October 26, 2007, the largest amount of gas consumption is obtained of all the cases listed compared to equal micro incremental method. The load dispatch results of unit $1 \#$ and $3 \#$ are 234.10MW and 265.26MW. The gas consumption is $42563.81 \mathrm{~m}^{3} \mathrm{~N} / \mathrm{h}$ and $43606.07 \mathrm{~m}^{3} \mathrm{~N} / \mathrm{h}$ respectively. The total gas consumption is $86169.88 \mathrm{~m}^{3} \mathrm{~N} / \mathrm{h}$, which has a reduction of $2337.45 \mathrm{~m}^{3} \mathrm{~N} / \mathrm{h}$ compared to equal micro incremental method and $1609.1 \mathrm{~m}^{3} \mathrm{~N} / \mathrm{h}$ compared to AGC instruction. It indicates that using MPGA to optimize the load dispatch problem of gas turbine units can improve the economic efficiency of the units.

\section{Conclusions}

Considering the importance of gas-fired generation in the field of energy, it is necessary to improve the efficiency of gas turbine power plants. Due to the complexity of gas turbine operation, the requirement for algorithms of good performance is strong.

Multiple population genetic algorithm is based on standard genetic algorithm and introduces multiple populations into optimal search process. In MPGA, different populations evolve simultaneously and are connected by the migration operator. The elite strategy can ensure message to be exchanged among all kinds of the populations and takes full advantage of knowledge accumulation in evolutionary process, so the solutions of the method are more reasonable.

By establishing fitness function, crossover, mutation and elite strategy, the theory of MPGA is used to build up a load dispatch model of the gas turbine generation units to optimize the load dispatch. Compared with the results of equal micro incremental method and AGC instruction, MPGA shows a decrease of gas consumption. Therefore, it indicates that MPGA has an advantage in the optimization of operation of gas turbine units. This work will contribute to the operation of gas turbine power plants.

\section{Acknowledgment}

The authors gratefully acknowledge the projects supported by "electric power research institute of China Southern Power Grid" and "the Fundamental Research Funds for the Central Universities” (Grant No.2009ZM0229and No. 2012ZM0015).

\section{REFERENCES}

[1] Abdolsaeid Ganjeh Kaviri, Mohammad Nazri Mohd. Jaafar, Tholudin Mat Lazim. "Modeling and Multi-objective Exergy Based Optimization of a Combined Cycle Power Plant Using A Genetic Algorithm,” Energy Conversion and Management, 2012, 58: 94-103.

[2] Narges Kaveshgar, Nathan Huynh, Saeed Khaleghi Rahimian. "An Efficient Genetic Algorithm for Solving the Quay Crane Scheduling Problem,” Expert Systems with Applications, 2012, 39: 13108-13117.

[3] Ma Xiaoqian, Wang Yi, Liao Yanfen. "Design and Realization of Power Plant Unit Load Optimization Scheduling Software System,”. Journal of South China University of Technology, 2006,34(6): 116-120.

[4] Huang Wenhua, Wang Renming, Liu Fen. "Hybrid Genetic Algorithm Applies on Power Plant Load Scheduling Problem," J of China Three Gorges Univ,2006,8(5): 400-403.

[5] Canforag, Dipentam, Espositor, Villaniml. "A Light Weight Approach for QoS-aware Service Composition,” Proc. of the 2th International Conference on Service Oriented Computing. New York, 2004, 232- 239

[6] Peng Xiangang, Zhang Conghui, Wang Xinghua. “Application Research of Optimal Load Dispatch on LNG Power Plant," Power System Protection and Control,2010,38(14): 84-87.

[7] Shi Feng, Wang Hui, Yu Lei. "30 Case Analysis of MATLAB Intelligent Algorithm,” Beijing: BEIHANG UNIVERSITY PRESS, 2011.

[8] Gong Jiawei, Chen Jun, Deng Haojiang. CA Selection "Method of Service Composition Based on Improved Genetic Algorithm," Micro Computer Applications, 2010 ,31(10): 1-6.

[9] Y. Toh, M. Koizumi, M. Oshima. "Analysis of toxic elements by MPGA," Journal of Radioanalytical and Nuclear Chemistry, 2010, 272 (2): 303-305.

[10] Wen Xianyang. “An improved genetic algorithm adopting immigration operator,” Intelligent Data Analysis, 2004 8:385-401.

[11] Wen H, Narula RG, "Economics of Gas Turbine Inlet Air Cooling for Combined Cycle Plants," Proceedings of the American power conference, Chicago, USA;2000. p. 100-105.

[12] Cheng Yang, Zeliang Yang, Ruixian Cai. “Analytical Method For Evaluation of Gas Turbine Inlet air Cooling in Combined Cycle Power Plant," Applied Energy 86 (2009) 848-856

[13] Jeffery K. Cochran, Shwu-Min Horng, John W. Fowler. "A Multi-population Genetic Algorithm to Solve Multi-objective Scheduling Problems for Parallel Machines," Computers \& Operations Research, June 2003, 30(7): 1087-1102.

[14] Y. G. Li, M. F. Abdul Ghafir, L. Wang. "Nonlinear Multiple Points Gas Turbine Off-Design Performance Adaptation Using a Genetic Algorithm,”. Journal of Engineering for Gas Turbines and Power, July 2011, Vol. 133. 